\title{
Structure of HIV-1 quasi-species as early indicator for switches of co-receptor tropism
}

\author{
J Nikolaj Dybowski, Dominik Heider, Daniel Hoffmann*
}

\begin{abstract}
Deep sequencing is able to generate a complete picture of the retroviral quasi-species in a patient. We demonstrate that the unprecedented power of deep sequencing in conjunction with computational data analysis has great potential for clinical diagnostics and basic research. Specifically, we analyzed longitudinal deep sequencing data from patients in a study with Vicriviroc, a drug that blocks the HIV-1 co-receptor CCR5. Sequences covered the V3-loop of gp120, known to be the main determinant of co-receptor tropism. First, we evaluated this data with a computational model for the interpretation of V3-sequences with respect to tropism, and we found complete agreement with results from phenotypic assays. Thus, the method could be applied in cases where phenotypic assays fail. Second, computational analysis led to the discovery of a characteristic pattern in the quasi-species that foreshadows switches of co-receptor tropism. This analysis could help to unravel the mechanism of tropism switches, and to predict these switches weeks to months before they can be detected by a phenotypic assay.
\end{abstract}

\section{Findings}

Human Immunodeficiency Virus 1 (HIV-1) enters cells in a complex process involving interactions of viral envelope protein gp120 with the cellular receptor CD4 and a co-receptor, typically one of the chemokine receptors CCR5 or CXCR4 [1]. According to their co-receptor usage or "tropism", viruses are classified as "R5" (interacting with CCR5) or "X4" (interacting with CXCR4). Additionally, there are dual-tropic "R5X4" strains that use both co-receptors for cell entry. Tropism is mainly determined by the sequence of the variable loop 3 (V3) of gp120. In initial infection, R5 viruses dominate the viral quasi-species [2]. As the disease progresses, about $50 \%$ of the patients develop X4 virus [3]. CCR5 blocking drugs, such as Maraviroc or Vicriviroc $[4,5]$ are ineffective against X4 virus, and thus it is advisable to test tropism prior to treatment with these drugs. The current state-of-the-art is testing by phenotypic assays such as Trofile ${ }^{\circ}$ (Monogram Biosciences, CA) [6] or enhanced sensitivity Trofile ${ }^{\bullet}$ assay (ESTA) [7]. However, their restriction to specialized laboratories, high cost and long turn-around are limiting availability. Moreover, phenotypic assays have been reported to fail in delivering any result in more than $15 \%$ of the

\footnotetext{
* Correspondence: daniel.hoffmann@uni-due.de

Department of Bioinformatics, Center for Medical Biotechnology, University of Duisburg-Essen, Universitätsstr. 1-5, D-45117 Essen, Germany
}

cases [8]. An alternative for routine diagnostics is genotypic testing: the genomic sequence of $\mathrm{V} 3$ from a patient is interpreted using computational models that relate V3 sequence and tropism. These models are typically derived by machine learning methods from a training set of V3 sequences and corresponding phenotypic test results [9-14]. Genotypic predictions can be made available via the Internet, and they are fast and cheap. Failure rates have been estimated to be around 7.5\% [8]. In clinical settings with tropism predictions based on single sequences from bulk sequencing, genotypic methods tend to perform less well [15], which is mostly attributed to low detection rates of $\mathrm{X} 4$ minorities by bulk sequencing [16]. Genotypic testing based on so-called "next generation sequencing" or "deep sequencing" methods may not suffer from this limitation [17] as they provide detailed data for the whole viral quasi-species. In fact, Vandenbroucke et al. [18] have demonstrated that a combination of deep sequencing of V3 with a genotype interpretation algorithm $[11,19]$ can be used for determination of tropism even in cases where phenotypic testing fails. In their study, the error rate of prediction methods was a limiting factor.

Recently, we have devised a two-level machine learning approach (T-CUP) [14] for the prediction of HIV-1 co-receptor usage from V3 sequences. At the first level, two independent predictions are made, based on the

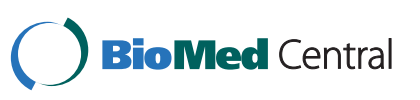

(c) 2010 Dybowski et al; licensee BioMed Central Ltd. This is an Open Access article distributed under the terms of the Creative Commons Attribution License (http://creativecommons.org/licenses/by/2.0), which permits unrestricted use, distribution, and reproduction in any medium, provided the original work is properly cited. 
electrostatic potential and hydropathy values [20] of the V3 loop, respectively. The predictions are then combined, and a final decision is reached. The method is accurate, provides predictions for all subtypes and is robust with respect to insertions and deletions. In this study, we applied T-CUP to deep sequencing data from Tsibris et al. [21], compared the predictions with results of phenotypic assays, and analyzed features of the viral quasi-species that could indicate tropism switches. Tsibris et al. had generated deep sequencing data for four patients at three time points during treatment with Vicriviroc, and concurrently had measured phenotypic tropism with the standard Trofile ${ }^{\bullet}$ assay. These assays had shown R5 virus at treatment start (Week 0 in Figure 1 and 2) for all patients. The common feature of all four patients was failure of Vicriviroc therapy.

We extracted V3 sequences in the viral quasi-species from the data published by Tsibris et al. by alignment to reference HXB2 V3 using Smith-Waterman local alignment [22] and translation into the corresponding amino acid sequence (Table 1). The tropism for every sequence in the quasi-species of each patient was predicted using T-CUP. The fraction of predicted X4 virus (i.e. number of predicted $\mathrm{X} 4$ tropic sequences divided by total number of sequences) in a quasi-species was compared with the outcome of the corresponding Trofile ${ }^{\circ}$ test. Figure 1 shows that there is a perfect agreement of the two Trofile ${ }^{\oplus}$ classes R5 and DM (dual/mixed) with predicted $\mathrm{X} 4$ tropic fractions of below and above 0.1, respectively. This agreement holds for all four patients and all time points, and is concordant with reports of

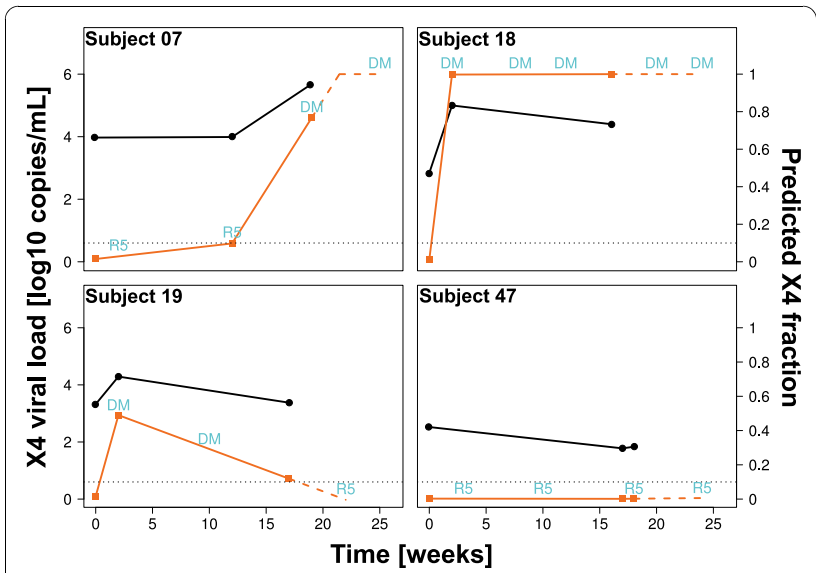

Figure 1 Development of fraction of X4 viruses. Development of predicted fraction of $\mathrm{X} 4$-using viruses during Vicriviroc treatment for four patients (right vertical axes, orange squares), and the contribution of that fraction to the absolute viral load (left vertical axes, black circles). Labels "R5" (R5-using) and "DM"(dual/mixed or X4-using) are Trofile ${ }^{\oplus}$ results at the given times. The dotted line marks the $10 \%$ detection rate of standard Trofile ${ }^{\circledast}$ assay. Naming was adopted from [21] reliable detection of $\mathrm{X} 4$ minorities in test mixtures by the standard Trofile ${ }^{\oplus}$ assay at concentrations down to $5-10 \%$ [6]. The phenotypic tropism assay has dichotomous output (either R5 or DM), while the T-CUP analysis of deep sequencing data generates a practically continuous (fraction of $\mathrm{X} 4$ tropic virus in quasi-species in units of $1 /$ (number of reads)). The latter allows for a more detailed characterization of the dynamics of the quasi-species with respect to tropism. It should be noted that the density of sampling points along the time axis in the Tsibris et al. dataset is too small for an accurate modeling of the tropism dynamics. However, the slopes of the lines in Figure 1 illustrate the principle. For instance, in the computational analysis of the quasispecies in Subject 07 we see an increase of the X4 fraction from week 0 over week 12 to week 19. From the slope between week 0 and week 12 we could extrapolate that shortly after week 12 a switch from R5 to DM should occur in the phenotypic assay. In fact, the phenotype data shows that the tropism switches between weeks 12 and 19 from R5 to DM. In the same way we would expect that the virus in Subject 18 remains DM tropic, and in Subject 47 R5 tropic. Subject 19 is a particularly interesting case with an early switch from R5 to $\mathrm{DM}$, accompanied by a steep increase in the $\mathrm{X} 4$ fraction according to T-CUP to 0.5 at week 2 . Then the X 4 fraction drops to slightly above 0.1 at week 17 . From this development we could extrapolate a reversion from DM to R5 shortly after week 17 (dashed blue line in Figure 1), as observed by Tsibris et al. [21].

We next exploited the property of T-CUP to provide in the first level two independent tropism predictions based on physical properties (electrostatics and hydropathy) of V3. The corresponding probabilities span a plane ("probabilities plane") in which every V3 sequence is represented by a point and the quasi-species by a cloud of such points. Figure 2 shows this plane for all twelve datasets from Ref. [21] with the points colored according to frequency of the respective sequence in the deep sequencing data.

The dynamics of the quasi-species in the probabilities plane has several remarkable features. First, all sequences in week 0 cluster in the lower left corner of the plane as is expected for a quasi-species that is R5 tropic. Second, the movement of the clouds indicates the dynamics of tropism. For Subjects 07 and 18 the clouds move towards the upper right, i.e. to more X4 tropism. For Subject 19 this movement is also seen for the first two time points but then reverts again to the lower left, i.e. to more R5 tropism. Subject 47 shows no marked movement to the upper right but remains localized in the lower left, in agreement with a quasi-species that remains R5 tropic. Third, for the patients 07,18 , and 19 where a co-receptor switch had been observed, 


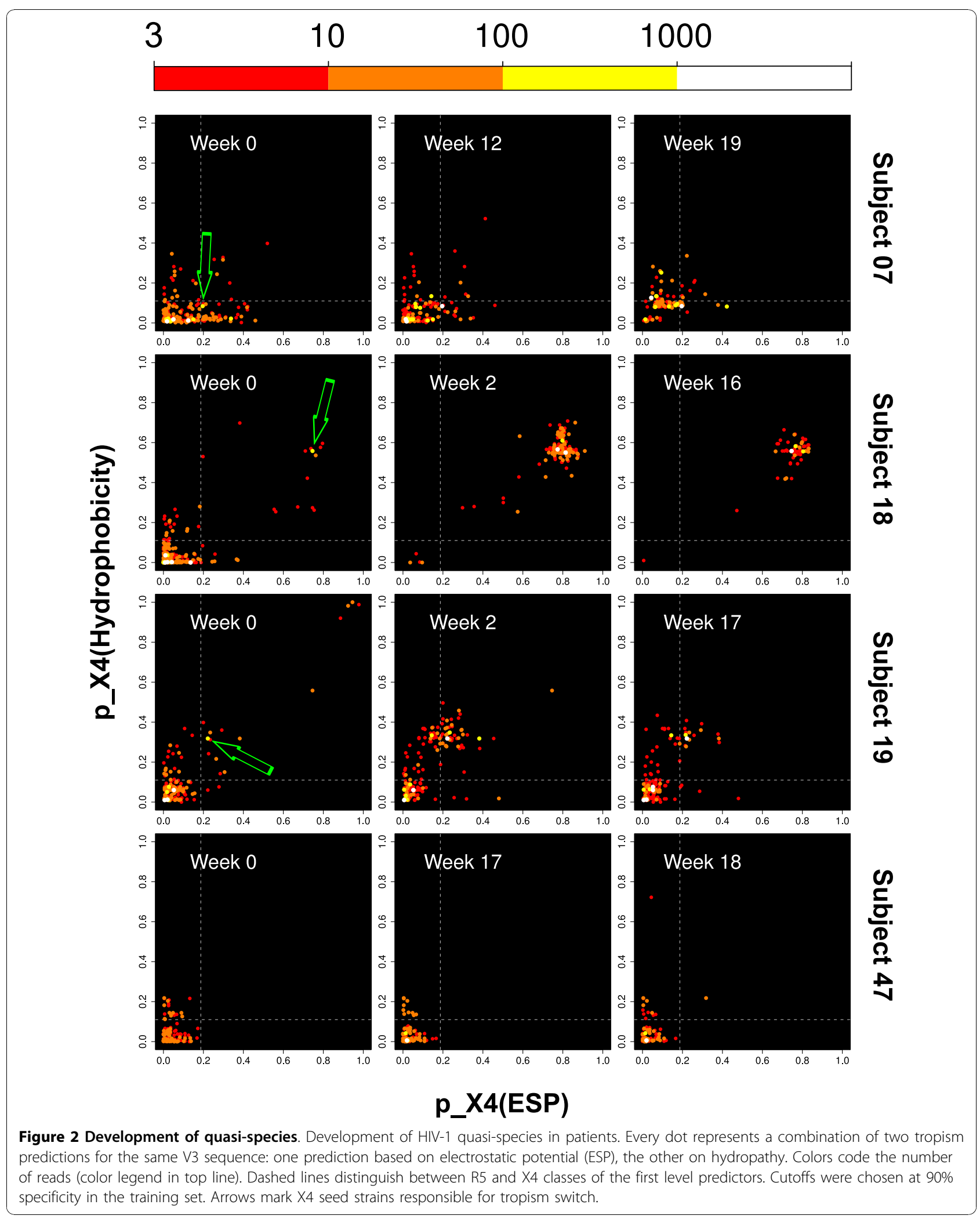


Table 1 Unique V3 sequences

\begin{tabular}{llll}
\hline Patient & time $\mathbf{1}$ & time $\mathbf{2}$ & time 3 \\
\hline 07 & 174 & 112 & 86 \\
18 & 240 & 112 & 41 \\
19 & 148 & 134 & 104 \\
47 & 126 & 84 & 78 \\
\hline
\end{tabular}

Number of unique sequences used in the analysis for each patient and each of the three sample times. Based on the data provided by Tsibris et al., a cutoff of a minimum of 4 reads per sequence was applied to limit the number of spurious sequences.

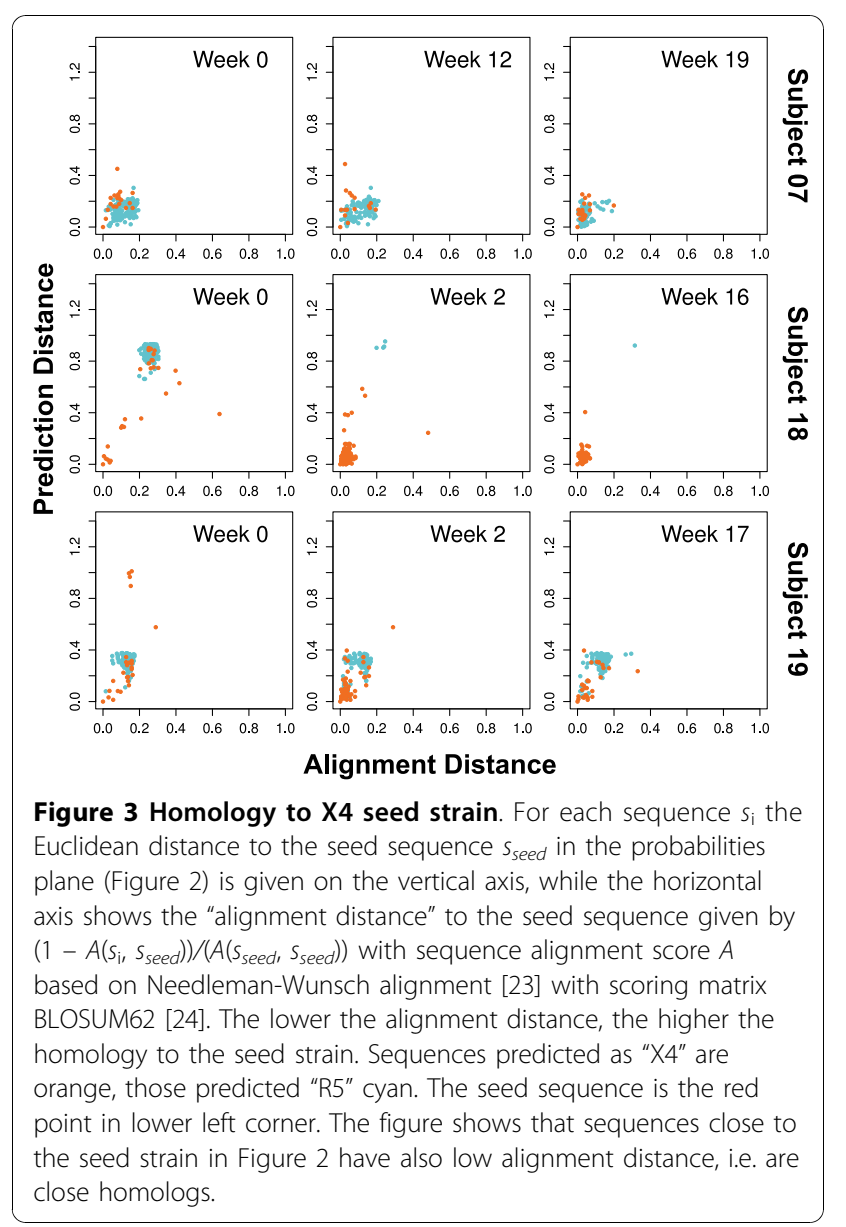

there is only one clearly dominating $\mathrm{X} 4$ strain in the probabilities plane, and this strain is already present at therapy start with considerable frequency (bright spots with green arrows). This "X4 seed strain" is specific for each of the patients - the seed strains for different patients are clearly located in different regions of the probabilities plane. Additionally, the $\mathrm{X} 4$ seed strain is accompanied from the beginning by a growing halo of local minor variants. Note that Subject 47 who remains R5 tropic throughout all time points does not have such a cluster.

We interpret the seed strain with its halo as a variant that has established itself in the quasi-species even at week 0 so that it can generate a considerable number of copies and also generates variant offspring. This interpretation is supported by the high homology of the sequences in the cluster (Figure 3). Using the statistical properties of this cluster in the probability plane (strong seed strain, halo of neighbor strains), it may become possible to predict a future co-receptor switch and therapy failure many weeks earlier than the switch of tropism becomes manifest in a phenotypic test. For such an early detection of a later switch of tropism, the resolution of deep sequencing and the accuracy of the prediction method is critical. For all three subjects where a switch occurs, the X4 seed strain initially accounts for $0.5 \%$ to $1 \%$ of the quasispecies (Table 2). The following progression towards $\mathrm{X} 4$ tropism is almost exclusively due to the expansion of these seed strains. For the three patients with a tropism switch a linear correlation of development of the total X4 fraction over time with the development of the fraction of the seed strain over time yields $R^{2}=$ $0.98\left(p=7.7 \cdot 10^{-10}\right)$.

Although the high cost of deep sequencing will probably prevent its use in routine diagnostics in the near future, the combination of this powerful method with accurate predictions could be applied when phenotype testing fails and to study evolution of viral quasi-species under selective pressure, and thus contribute to the development of sustainably effective treatments.

Table 2 Development of $\mathrm{X} 4$ variants causing tropism switch

\begin{tabular}{|c|c|c|c|c|}
\hline \multirow[t]{2}{*}{ Patient } & \multirow[t]{2}{*}{ Variant } & \multicolumn{3}{|c|}{ Fraction of population at } \\
\hline & & time 1 & time 2 & time 3 \\
\hline 07 & CTRPGNNTRRSIRIGPGQTFFAREDI IGDIRQAYC & 0.01 & 0.07 & 0.73 \\
\hline 18 & CERPNNNTRQRLSIGPGRSFYTSRRI IGDVKKAHC & 0.005 & 0.79 & 0.71 \\
\hline 19 & CTRPNNNTRKGIYLGPGRAFYTTDKI IGDIRQAHC & 0.007 & 0.43 & 0.08 \\
\hline 47 & CTRPNNSTRKS INIGPGSAWYTTGDI IGDIRQAHC & 0.0009 & 0.0 & 0.0 \\
\hline
\end{tabular}

Development of the $\mathrm{X} 4$ seed strains in Subjects $07,18,19$ with tropism switches and the largest initial X4 strain in patient 47 who does not show a tropism switch. The three times are the sampling points along the "Time" axis in Figure 1. 


\section{Acknowledgements}

This work was funded by BMBF grant $01 E S 0709$ and DFG TRR 60/A6. The authors thank Hauke Walter for fruitful discussions, and Tsibris et al. [21] for making their data available to the public.

\section{Authors' contributions}

JND devised and carried out the research, analyzed data, and drafted the manuscript. D Heider contributed to data analysis and to drafting of the manuscript. D Hoffmann has devised research, analyzed data, and revised the manuscript. All authors read and approved the final manuscript.

\section{Competing interests}

The authors declare that they have no competing interests.

Received: 22 September 2010 Accepted: 30 November 2010

Published: 30 November 2010

\section{References}

1. D'Souza MP, Harden VA: Chemokines and HIV-1 second receptors. Confluence of two fields generates optimism in AIDS research. Nat Med 1996, 2(12):1293-1300

2. Connor RI, Sheridan KE, Ceradini D, Choe S, Landau NR: Change in coreceptor use correlates with disease progression in HIV-1-infected individuals. J Exp Med 1997, 185(4):621-628.

3. Berger EA, Murphy PM, Farber JM: Chemokine receptors as HIV-1 coreceptors: roles in viral entry, tropism, and disease. Annu Rev Immunol 1999, 17:657-700.

4. Dorr P, Westby M, Dobbs S, Griffin P, Irvine B, Macartney M, Mori J, Rickett G, Smith-Burchnell C, Napier C, Webster R, Armour D, Price D, Stammen B, Wood A, Perros M: Maraviroc (UK-427,857), a potent, orally bioavailable, and selective small-molecule inhibitor of chemokine receptor CCR5 with broad-spectrum anti-human immunodeficiency virus type 1 activity. Antimicrob Agents Chemother 2005, 49(11):4721-4732.

5. Strizki JM, Tremblay C, Xu S, Wojcik L, Wagner N, Gonsiorek W, Hipkin RW, Chou CC, Pugliese-Sivo C, Xiao Y, Tagat JR, Cox K, Priestley T, Sorota S, Huang W, Hirsch M, Reyes GR, Baroudy BM: Discovery and characterization of vicriviroc (SCH 417690), a CCR5 antagonist with potent activity against human immunodeficiency virus type 1 . Antimicrob Agents Chemother 2005, 49(12):4911-4919.

6. Whitcomb JM, Huang W, Fransen S, Limoli K, Toma J, Wrin T, Chappey C, Kiss LDB, Paxinos EE, Petropoulos CJ: Development and characterization of a novel single-cycle recombinant-virus assay to determine human immunodeficiency virus type 1 coreceptor tropism. Antimicrob Agents Chemother 2007, 51(2):566-575.

7. Saag M, Heera J, Goodrich J, DeJesus E, Clumeck N, Cooper D, Walmsley S, Ting N, Coakley E, Reeves J, Westby M, van der Ryst E, Mayer H: Reanalysis of the MERIT study with the enhanced Trofile assay (MERIT-ES). 48th Interscience Conference on Antimicrobial Agents and Chemotherapy, Washington, DC 2008.

8. Poveda E, Seclen E, del Mar González M, García F, Chueca N, Aguilera A, Rodríguez JJ, Gonzalez-Lahoz J, Soriano V: Design and validation of new genotypic tools for easy and reliable estimation of HIV tropism before using CCR5 antagonists. J Antimicrob Chemother 2009, 63(5):1006-1010.

9. Resch W, Hoffman N, Swanstrom R: Improved success of phenotype prediction of the human immunodeficiency virus type 1 from envelope variable loop 3 sequence using neural networks. Virology 2001, 288:51-62.

10. Pillai $S$, Good B, Richman D, Corbeil J: A new perspective on V3 phenotype prediction. AIDS Res Hum Retroviruses 2003, 19(2):145-149.

11. Jensen MA, Li FS, van't Wout AB, Nickle DC, Shriner D, He HX McLaughlin S, Shankarappa R, Margolick JB, Mullins JI: Improved coreceptor usage prediction and genotypic monitoring of R5-to-X4 transition by motif analysis of human immunodeficiency virus type 1 env V3 loop sequences. J Virol 2003, 77(24):13376-13388.

12. Sander $O$, Sing $T$, Sommer I, Low AJ, Cheung PK, Harrigan PR, Lengauer $T$, Domingues FS: Structural descriptors of gp120 V3 loop for the prediction of HIV-1 coreceptor usage. PLoS Comput Biol 2007, 3(3):e58.

13. Xu S, Huang $X, X u H$, Zhang C: Improved prediction of coreceptor usage and phenotype of HIV-1 based on combined features of V3 loop sequence using random forest. J Microbiol 2007, 45(5):441-446.

14. Dybowski JN, Heider D, Hoffmann D: Prediction of Co-Receptor Usage of HIV-1 from Genotype. PLoS Computational Biology 2010, 6(4):e1000743.
15. Low A, Dong W, Chan D, Sing T, Swanstrom R, Jensen M, Pillai S, Good B, Harrigan P: Current V3 genotyping algorithms are inadequate for predicting X4 co-receptor usage in clinical isolates. Aids 2007, 21(14):F17.

16. Skrabal K, Low AJ, Dong W, Sing T, Cheung PK, Mammano F, Harrigan PR: Determining human immunodeficiency virus coreceptor use in a clinical setting: degree of correlation between two phenotypic assays and a bioinformatic model. J Clin Microbiol 2007, 45(2):279-284

17. Swenson L, Dong W, Mo T, Woods C, Thielen A, Jensen M, Glascock C, Montaner J, Harrigan R: Quantification of HIV tropism by "deep" sequencing shows a broad distribution of prevalence of X4 variants in clinical samples that is associated with virological outcome. 16th Conference on Retroviruses and Opportunistic Infections, Montreal, Canada 2009:Poster 680.

18. Vandenbroucke I, Marck HV, Mostmans W, Eygen W, Rondelez E, Thys K, Baelen KV, Fransen K, Vaira D, Kabeya K, Wit SD, Florence E, Moutschen M, Vandekerckhove L, Verhofstede C, Stuyver LJ: HIV-1 V3 envelope deep sequencing for clinical plasma specimens failing in phenotypic tropism assays. AIDS Research and Therapy 2010, 7:4

19. Sing T, Low AJ, Beerenwinkel N, Sander O, Cheung PK, Domingues FS, Büch J, Däumer M, Kaiser R, Lengauer T, Harrigan PR: Predicting HIV coreceptor usage on the basis of genetic and clinical covariates. Antivir Ther 2007, 12(7):1097-1106.

20. Kyte J, Doolittle R: A simple method for displaying the hydropathic character of a protein. J Mol Biol 1982, 157:105-132.

21. Tsibris AMN, Korber B, Arnaout R, Russ C, Lo CC, Leitner T, Gaschen B, Theiler J, Paredes R, Su Z, Hughes MD, Gulick RM, Greaves W, Coakley E, Flexner C, Nusbaum C, Kuritzkes DR: Quantitative deep sequencing reveals dynamic HIV-1 escape and large population shifts during CCR5 antagonist therapy in vivo. PLoS One 2009, 4(5):e5683.

22. Smith TF, Waterman MS: Identification of common molecular subsequences. J Mol Biol 1981, 147:195-197.

23. Needleman S, Wunsch C: A general method applicable to the search for similarities in the amino acid sequence of two proteins. Journal of molecular biology 1970, 48(3):443-453.

24. Heniko S, Heniko J: Amino acid substitution matrices from protein blocks Proceedings of the National Academy of Sciences of the United States of America 1992, 89(22):10915.

doi:10.1186/1742-6405-7-41

Cite this article as: Dybowski et al: Structure of HIV-1 quasi-species as early indicator for switches of co-receptor tropism. AIDS Research and Therapy 2010 7:41.

\section{Submit your next manuscript to BioMed Central and take full advantage of:}

- Convenient online submission

- Thorough peer review

- No space constraints or color figure charges

- Immediate publication on acceptance

- Inclusion in PubMed, CAS, Scopus and Google Scholar

- Research which is freely available for redistribution
Biomed Central 